\title{
Hydrodynamique et transfert de matière dans un contacteur gaz-liquide multicellulaire
}

\author{
Hydrodynamics and mass transfer \\ in a multicellular gas-liquid contactor
}

\author{
Primius Boldo*, André Laurent*, \\ Christian Belin** et Jean-Claude Charpentier* \\ ${ }^{*}$ Laboratoire des Sciences du Génie Chimique (Nancy) \\ ${ }^{*}$ Europoll - Techfina Engenierie
}

\section{Introduction}

L'objectif principal de l'étude consiste à définir globalement les caractéristiques d'un nouveau laveur gazliquide original conçu par Europoll et commercialisé par Techfina et Europoll, dont l'application essentielle réside dans la lutte contre la pollution chimique et particulaire de l'air.

Les résultats rapportés ici constituent une première approche du fonctionnement du laveur multicellulaire Techfina sous les aspects de l'hydrodynamique et du transfert de matière.

La finalité de cette première étude doit permettre également d'orienter le programme ultérieur de recherche de façon à appréhender tous les problèmes relatifs à l'exploitation du laveur multicellulaire et de le comparer aux autres réacteurs gaz-liquidc classiques.

\section{Description de l'appareil}

Le laveur multicellulaire Techfina est un nouveau contacteur gaz-liquide constitué par un empilement régulier selon les trois axes de cellules ou motifs élémentaires dans lesquels le gaz et le liquide circulent verticalement à contre courant.

Le laveur multicellulaire est constitué de trois parties principales:

- une trémie inférieure d'entrée des gaz et de récupération du liquide de lavage,

- un corps modulaire de lavage des gaz (fig. 1).

- une coiffe de sortie de gaz traités et d'entrée et distribution du liquide de lavage.

(*) Laboratoire des Sciences du Génie Chimique. CNRS. ENSIC. 1 Rue Granville - 54042 Nancy

(**) Europoll, 2 rue Amorteaux, 78730 St-Arnoult en Yvelines. Techfina Engenierie

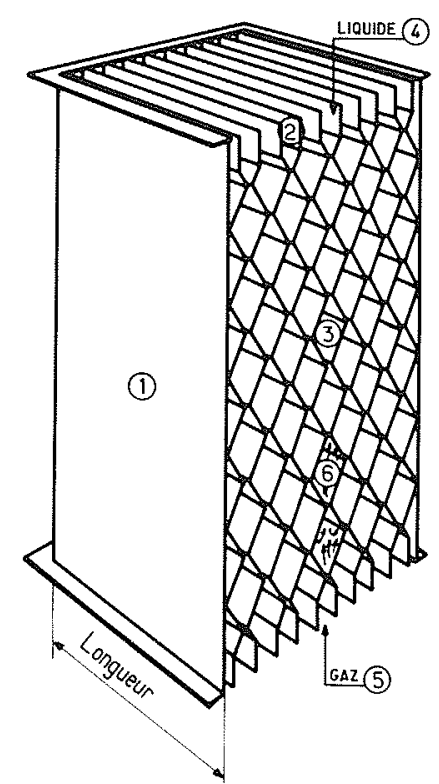

Figure 1. - Laveur multicellulaire.

Le corps est constitué d'une enveloppe simple verticale (1). Dans cette enveloppe sont glissés verticalement des panneaux (2) munis de motifs horizontaux répétitifs (3).

Le liquide de lavage est déversé par le haut des panneaux (4). Les gaz sont traités de bas en haut entre les panneaux (5).

A chaque motif, les gaz sont lavés successivement par un barbotage des liquides (6).

La figure 2 montre un motif élémentaire classique constitué d'un convergent et d'un divergent de section rectangulaire dont l'intersection constitue le col. Le motif élémentaire est défini par la largeur e du col, la 


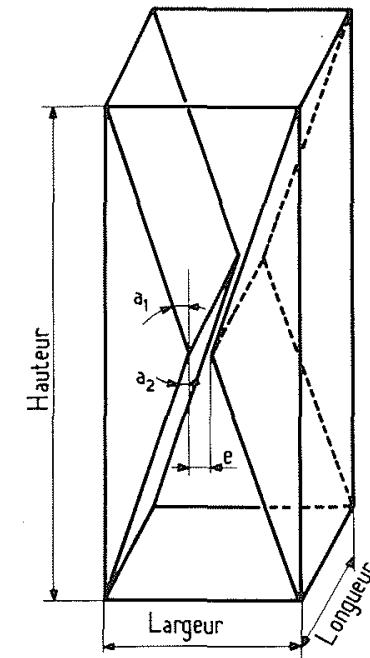

Figure 2. - Motif élémentaire

forme du col, les angles du convergent et du divergent, la hauteur, la largeur et la longueur.

La présente étude rapporte les résultats obtenus lors de l'expérimentation d'un motif élémentaire de $10 \mathrm{~mm}$ de largeur de col, de $200 \mathrm{~mm}$ de hauteur, de $80 \mathrm{~mm}$ de largeur et de $80 \mathrm{~mm}$ de longueur et d'un empilement de plusieurs de ces motifs ( $n=1$ à 9 ).

\section{Hydrodynamique du laveur}

L'étude des écoulements diphasiques en maquette froide (air-eau) du laveur Techfina a pour buts de décrire les différents régimes hydrodynamiques d'écoulement des phases, de déterminer les pertes de charge subies par le gaz en écoulement et de mesurer les rétentions de liquide dans le laveur. La synthèse des résultats permet d'en déduire le diagramme de fonctionnement du laveur.

\section{Régimes d'écoulement}

L'observation visuelle du mode d'écoulement des phases et l'examen de la variation des paramètres hydrodynamiques (perte de charge et rétention) permettent de définur différents régimes d'écoulement.

\section{Régime ruisselant}

Lorsque les débits gazeux (ou) et liquide sont faibles, il n'y a pas d'interaction entre les deux fluides. Chacun s'écoule comme s'il était seul. Le liquide peut notamment s'écouler soit sous forme de "rivières" ou "torrents", soit sous forme de film, suivant l'état préalable de la surface de l'appareil.

\section{Régime de vagues}

Lorsque la perte de charge augmente, du fait soit d'une augmentation du débit de gaz, soit d'un accroissement du débit de liquide, on passe pendant une courte période par un régime de vagues. Il apparaît ici une interaction entre le gaz et le liquide sous la forme de vagues à la surface du liquide.

\section{Zone d'accrochage}

L'accrochage est l'instant où les vagues du régime précédent se rejoignent au col. Ce phénomène se traduit par une brutale augmentation simultanée de la perte de charge et de la rétention.

Dans un empilement de motifs élémentaires, l'accrochage peut se produire autant de fois qu'il y a de motifs individuels.

La zone de transition à l'accrochage est d'autant plus importante que le nombre de motifs est élevé.

L'accrochage complet d'un empilement de motifs identiques est toujours obtenu à débit de liquide donné pour le même débit gazeux quel que soit le nombre de motifs.

\section{Engorgement}

L'engorgement d'un motif unique est caractérisé par l'accumulation de liquide à son sommet.

Ce phénomène a lieu pour une perte de charge sensiblement constante, quels que soient les débits gazeux et liquide traversant le motif considéré.

Dans un empilement de motifs, ce phénomène est toujours caractérisé par l'engorgement du motif supérieur et un assèchement progressif des autres motifs inférieurs quel que soit le nombre de motifs.

\section{Zone de fonctionnement}

La zone de fonctionnement est définie, dans le système d'axes débit de gaz versus débit de liquide, par l'ensemble des couples de débits du domaine limité par

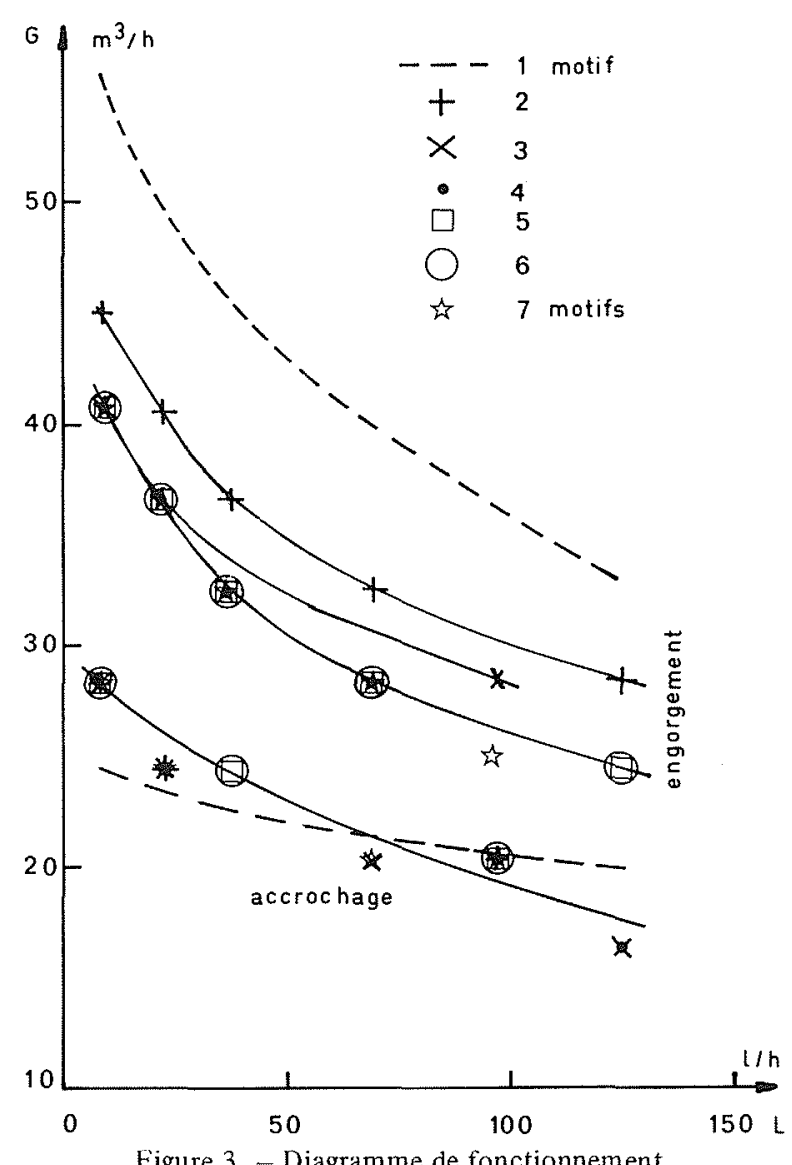


les phénomènes respectifs d'accrochage et d'engorgement. C'est la zone d'utilisation stable.

Cette zone de stabilité correspond à l'utilisation fonctionnelle du laveur Techfina en tant que réacteur gazliquide.

La figure 3 présente le diagramme de fonctionnement d'un laveur multicellulaire, où il est possible de se rendre compte de l'influence du nombre de motifs constituant l'empilement.

On constate en effet, d'une part que la courbe d'accrochage est pratiquement indépendante du nombre de motifs, comme cela a d'ailleurs été indiqué lors de la des. cription du phénomène d'accrochage.

On observe, d'autre part un étagement de la limite supérieure du fonctionnement à l'engorgement.

La conséquence qui en résulte se traduit par une réduction de la plage de fonctionnement stable du laveur en fonction du nombre croissant de motifs de l'empilement. Il semble toutefois que cette diminution tende vers une limite au-delà de quatre motifs.

\section{Perte de charge}

La figure 4 présente un exemple de l'évolution de la perte de charge dans un motif élémentaire en fonction du débit de gaz pour différents débits de liquide.

Le point essentiel est que ces courbes sont discontinues. Pour un débit de liquide donné, la première partie de la courbe, obtenue en augmentant progressivement le débit de gaz, représente le fonctionnement du motif avant accrochage en régime ruisselant ou de vagues. La seconde partie de la courbe, non raccordée à la première, correspondant à des débits de gaz encore plus importants, rend compte de la variation de la perte de charge dans la zone de fonctionnement comprise entre l'accrochage et l'engorgement.

Il existe entre les deux parties une zone de transition dans laquelle il est difficile de décrire le passage ou le saut brutal conduisant à l'accrochage.
Notons par ailleurs que l'allure des courbes de pertes de charge obtenues dans un empilement de motifs élémentaires est identique [1].

Il est possible de prévoir la perte de charge totale dans un empilement de motifs à partir des résultats obtenus sur un motif élémentaire. En effet, l'additivité des pertes de charge en fonction du nombre de motifs a été établie dans un domaine correspondant à une compatibilité de fonctionnement indépendante de la configuration de l'empilement, ceci afin de tenir compte de l'incidence du nombre de motifs sur la définition de la zone de fonctionnement [1].

\section{Rétentions de liquide}

La rétention de liquide est le volume de liquide contenu dans le laveur en régime permanent de fonctionnement. Elle est exprimée soit en volume effectif de liquide, soit en pourcentage du volume total du laveur.

Cette grandeur est déterminée par la technique de collection de liquide en pied de l'appareil après arrêt simultané des débits de liquide ou de gaz.

L'allure de la variation de la rétention en fonction du débit de gaz pour un débit de liquide fixé est identique à celle de l'évolution des pertes de charge où le phénomène d'accrochage provoque ici aussi une discontinuité. Cette identité de variation reste encore vraie dans le cas d'un empilement de motifs.

Enfin l'additivité de la grandeur rétention de liquide en fonction du nombre de motifs a été également vérifiée [1].

\section{Relation entre la perte de charge et la rétention}

La similitude des variations des pertes de charge et des rétentions en fonction du débit gazeux conduit naturellement à essayer de relier ces deux grandeurs. Une solution satisfaisante consiste à présenter la rétention en fonction de la perte de charge rapportée à une

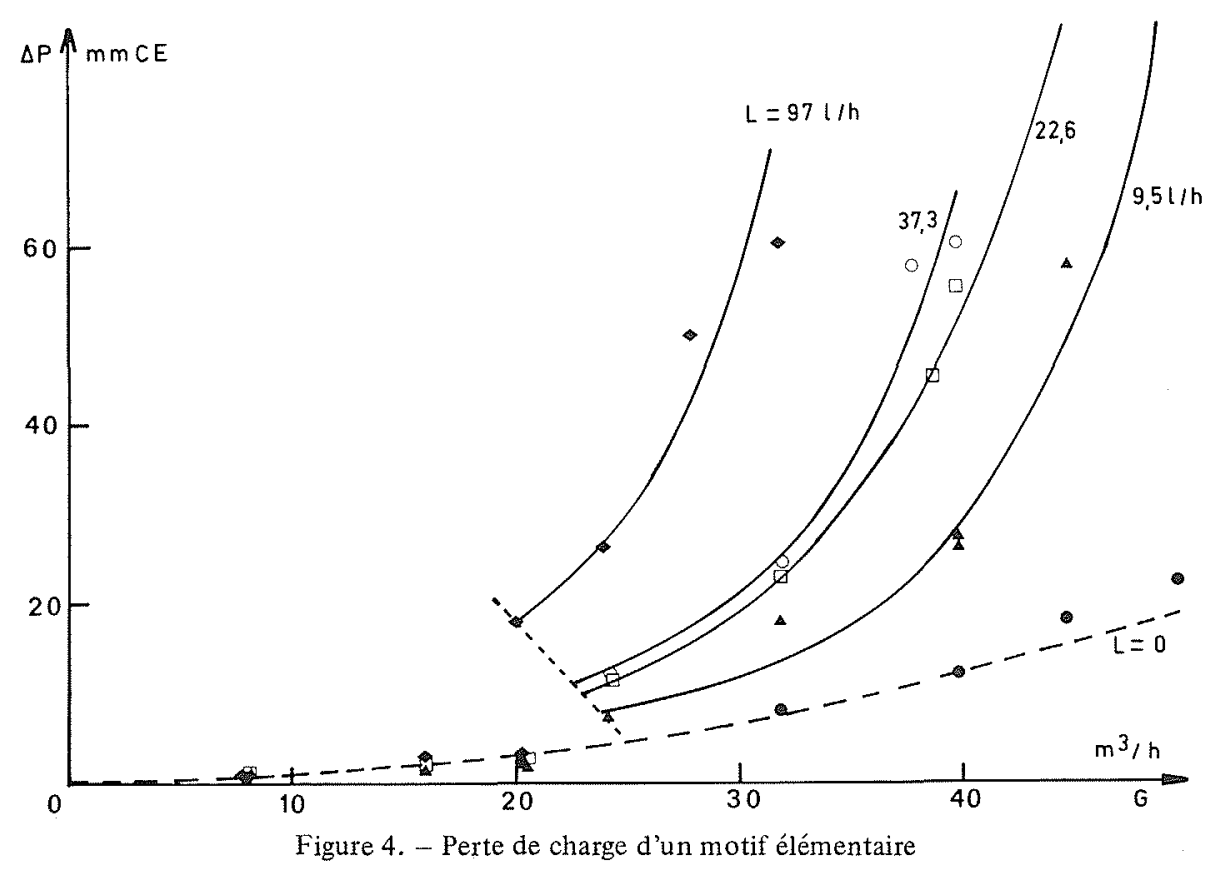




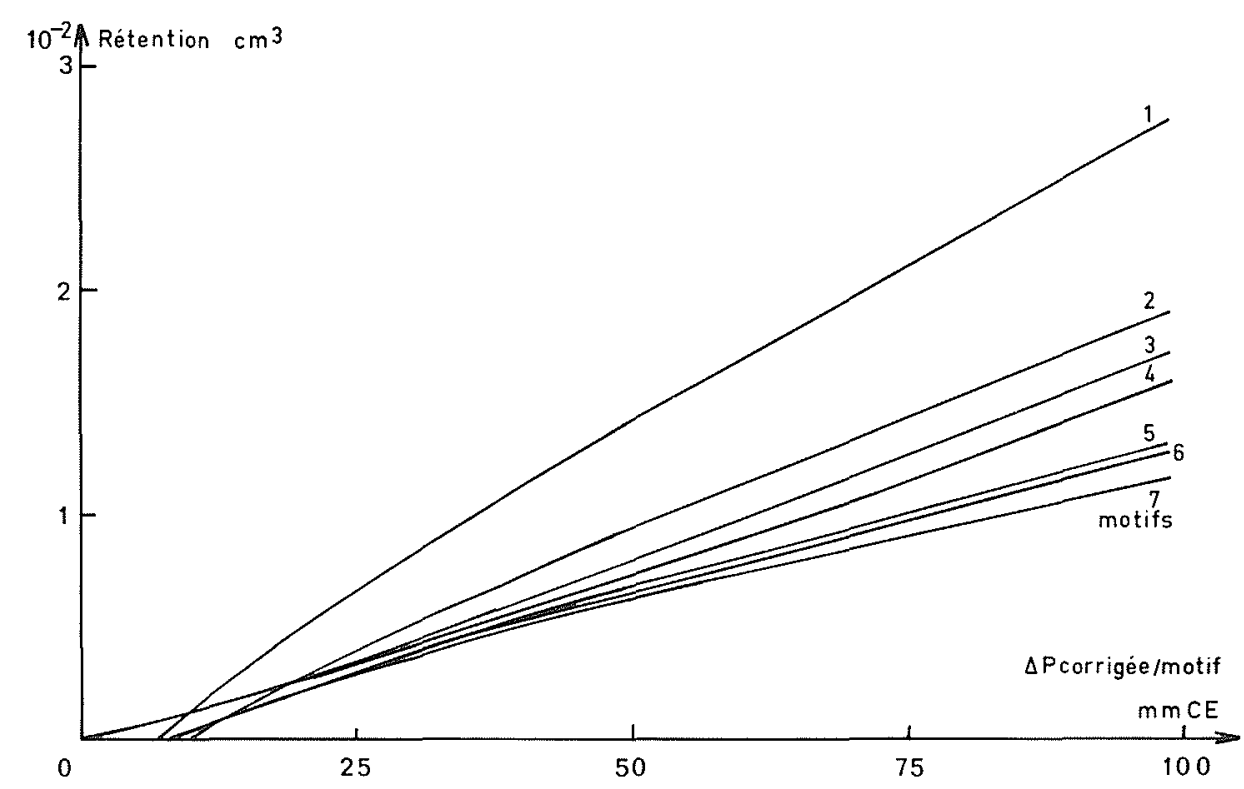

Figure 5. - Variation de la rétention de liquide en fonction de la perte de charge corrigée par motif et du nombre de motifs.

même vitesse de gaz au col du motif, que nous appellerons "perte de charge corrigée".

Cette notion de perte de charge corrigée permet de rendre compte de façon satisfaisante de l'ensemble des résultats expérimentaux obtenus quelle que soit la configuration du laveur.

La figure 5 illustre cette représentation, qu'il est possible d'assimiler sensiblement à un faisceau de droites. Une interprétation possible consiste à supposer qu'une fraction constante de la perte de charge ne crée pas de rétention (effets de bout et perte de charge à sec) et qu'une autre partie de la perte de charge est consommée pour produire le primage des motifs. Cette dernière quantité, étant d'autant plus grande que le nombre de motifs est important, provoque ainsi l'apparition d'un faisceau de droites à pente dégressive.

\section{Transfert de matière}

L'objectif de l'étude est d'essayer de caractériser globalement le laveur multicellulaire Techfina du point de vue transfert de matière.

Dans ce but l'absorption du dioxyde de soufre dilué par l'air dans une solution aqueuse d'hydroxyde de sodium a été mise en œuvre dans les conditions d'une réaction irréversible, instantanée, de surface, et entièrement contrôlée par la diffusion du soluté dans la phase gazeuse.

Les essais consistent simplement à mesurer simultanément pour un couple donné de débits liquide et gazeux les concentrations du soluté gazeux à l'entrée et à la sortie du (ou des) motif(s) et la perte de charge totale.

Les performances du laveur sont alors exprimées pour ce système chimique par la valeur de l'efficacité ou celle du nombre d'unités de transfert en phase gazeuse en appliquant les relations

$E_{G}=\left(y_{E}-y_{S}\right) / y_{E}$

$N_{G}=\ln \left(y_{E} / y_{S}\right)=-\ln \left(1-E_{G}\right)$

La figure 6 présente la variation de l'efficacité globale obtenue dans le motif étudié en fonction de la perte de charge subie par le gaz. L'allure générale de la figure rend compte de l'évolution classique de l'efficacité, dont la valeur est d'autant plus importante que le débit de liquide est élevé et que le débit de gaz est faible.

Remarquons que dans le domaine hydrodynamique habituel de fonctionnement et pour le système étudié, l'ordre de grandeur de l'efficacité est de 50 à $60 \%$.

Signalons cependant que la grandeur efficacité tend vers une limite asymptotique quel que soit le

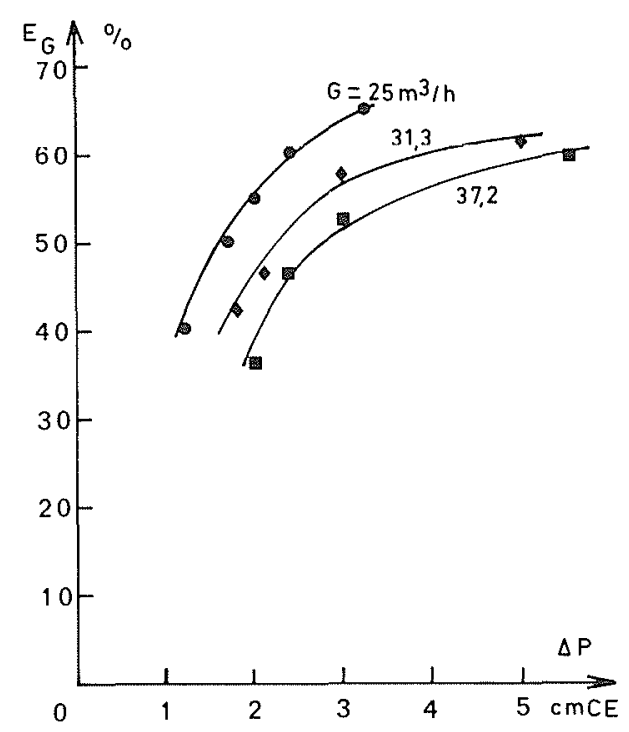

Figure 6. - Efficacité d'un motif en fonction de la perte de charge. 


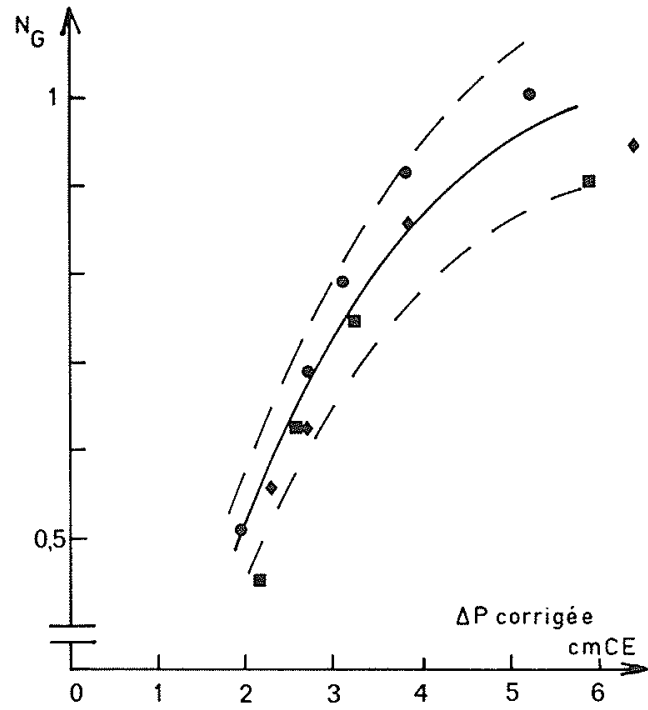

Figure 7. - Variation du nombre d'unités de transfert d'un motif en fonction de la perte de charge corrigée.

débit de gaz quand le fonctionnement du motif s'approche de la zone d'engorgement [1].

L'étude hydrodynamique a montré que la notion de perte de charge corrigée, c'est-à-dire ramenée à une même vitesse de gaz au col, permet de relier globalement la rétention et la perte de charge des motifs.

Cette notion est reprise ici pour tenter de relier de la même façon le transfert de matière à l'hydrodynamique.

La figure 7 rapporte les variations du nombre global d'unités de transfert en phase gazeuse en fonction de la perte de charge corrigée quel que soit le débit de gaz dans le motif. L'ensemble des résultats est bien représenté autour de la courbe moyenne avec une dispersion de $\pm 10 \%$ dans le domaine de fonctionnement stable du motif.

Le fait d'empiler les motifs élémentaires est une démarche essentielle de la possibilité d'extrapolation en hauteur du laveur multicellulaire Techfina. Il importe donc de se préoccuper de ce que deviennent les résultats de transfert de matière acquis sur un motif élémentaire lors du passage à un empilement de motifs.

Les résultats de transfert de matière, obtenus dans une zone d'utilisation pratique des divers empilements comprise entre l'accrochage et $60 \%$ de l'engorgement, sont reportés sur la figure 8 en adoptant la présentation $N_{G}$ versus perte de charge corrigée

Ces résultats montrent d'une part, à débit de gaz constant, que les performances d'un empilement s'amenuisent au fur et à mesure que le nombre de motifs élémentaires le constituant augmente et que le gain potentiel d'efficacité dû à la multiplication du nombre de modules reste limité pour le système étudié. D'autre part, cet effet paraît d'autant plus net que le débit de gaz traversant le laveur est élevé.

Un phénomène global identique a déjà été observé lors de la caractérisation des performances des dépoussiéreurs. La littérature rapporte en effet l'existence d'un tassement progressif du nombre d'unités de transfert matérialisant l'efficacité de capture des particules d'un dépoussiéreur malgré l'augmentation progressive de la perte de charge dégradée. Il semble alors que des mécanismes différents de capture puissent expliquer ce comportement [2].

Dans le cas du transfert de matière dans le laveur multicellulaire, il est probable que la non-additivité des motifs résulte du primage des motifs, ce dernier étant d'autant plus intense que le débit de gaz est important.

\section{Conclusion}

Cette étude exploratoire ne constitue qu'une première étape de dégrossissage du fonctionnement du laveur gaz-liquide de type nouveau que constitue l'appareil Techfina.

Les aspects relatifs à l'hydrodynamique et au transfert de matière mettent clairement en évidence la nécessité d'étudier non seulement le motif élémentaire, mais aussi l'empilement de cellules élémentaires avant de

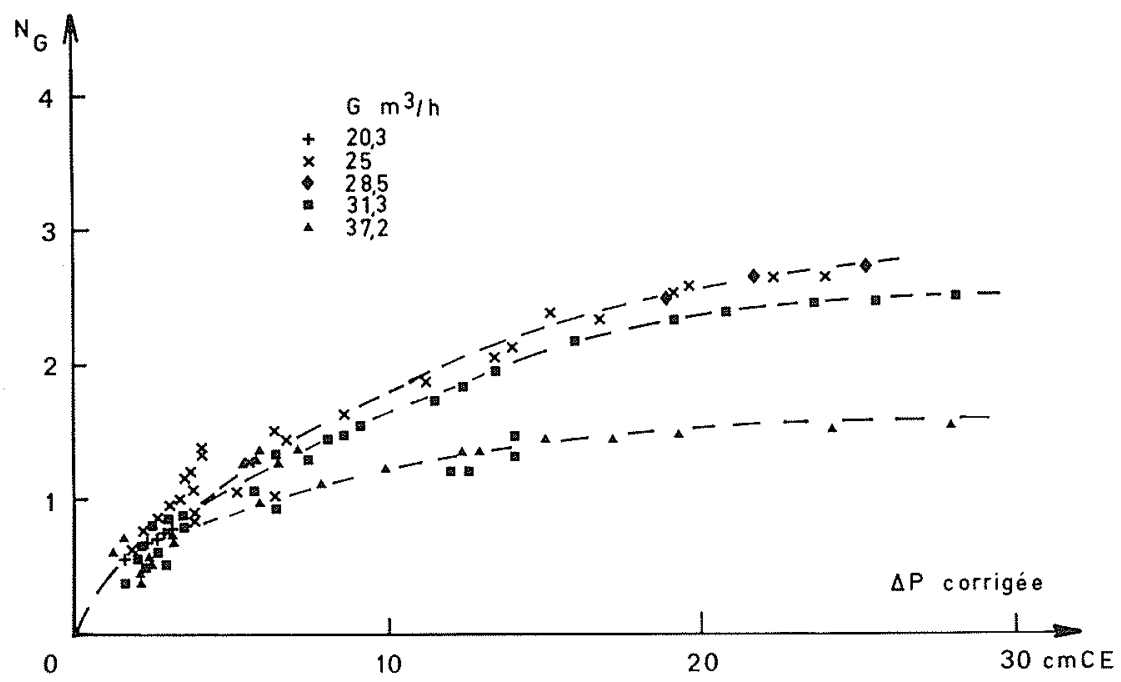

Figure 8. - Transfert de matière dans un empilement de motifs. 
tenter l'extrapolation des performances d'un laveur industriel.

Une vue prospective de cette possibilité séduisante d'extrapolation doit également prendre en compte l'influence certaine de la longueur du col des motifs, dont l'examen n'a pas été abordé quantitativement dans ce travail.

Enfin le laveur doit être caractérisé plus finement du point de vue transfert de matière en précisant les valeurs des conductances de chacune des phases et de l'aire interfaciale par mise en œuvre de la méthodologie maintenant classique de l'absorption gaz-liquide avec réaction chimique.

\section{Nomenclature}

$a_{1} \quad$ angle du divergent d'un motif

$a_{2}$ angle du convergent d'un motif e largeur du col d'un motif

$E_{G} \quad$ efficacité globale en phase gazeuse

$G$ débit volumique de gaz

$L$ débit volumique de liquide

$n$ nombre de motifs

$N_{G} \quad$ nombre d'unités de transfert en phase gazeuse

$\triangle P$ perte de charge d'un motif ou d'un empilement

$y_{E} \quad$ titre molaire en soluté à l'entrée du laveur

$y_{S}$ titre molaire en soluté à la sortie du laveur.

\section{Références bibliographiques}

[1]BOLDO P. - Hydrodynamique et transfert de matière dans un type nouveau de réacteur gaz-liquide : le laveur de gaz multicellulaire. Paramètres fondamentaux. Domaines de fonctionnement. Thèse CNAM, Nancy 1979.

[2] SEMRAU K.T. - Practical process design of particulate scrubbers. Chemical Engineering, 1977, Septem ber 26, 87-91.

\section{Discussion}

Président : M. P. TRAMBOUZE

Le Président. - Je remercie M. BELIN de son exposé si judicieusement assorti d'une démonstration sur maquette et de la projection d'un film. Il s'agit d'un travail fait en collaboration entre la Société TECHFINA, qui est le fabricant de l'appareil dont vous avez eu la démonstration, et de l'équipe de $M$. CHARPENTIER au Laboratoire des Sciences du Génie Chimique à Nancy. C'est la réalisation de l'intégration Université - C.N.R.S. - Industrie qui est tant recherchée à l'heure actuelle.

M. DELHAYE. - A-t-on essayé de corréler les limites d'engorgement et les limites d'accrochage en fonction des débits de liquide et de gaz et des propriétés physiques de ces fluides ? $\mathrm{Si}$ oui, quelle est la longueur caractéristique qui joue le tôle de facteur d'échelle?

M. BELIN. - L'engorgement est le régime de marche de l'appareil, c'est un reflux contrôlé.

Au-dessous de ce régime, il existe une cassure très rapide que l'on nomme accrochage. Il serait très intéressant de vérifier dans cette zone, vis-à-vis de l'utilisation maximum de l'énergie, si cette cassure représente quelque chose, et pouvoir obtenir l'accrochage.

Par contre, la notion de densité du liquide va jover. Il est certain que, s'il y a énormément de dynamique, toute l'énergic se situe dans la quantité de mouvement communiquée aux gouttes.

En revanche, au niveau de la pression statique, qui va faire bouger la masse de liquide grâce à l'énergie apportée par les gaz, c'est là, à mon sens, qu'est l'apport important, celui de la turbulence au niveau de cette aire interfaciale qui est en train de se créer. Là encore, il existe un champ de recherches important pour savoir comment utiliser le mieux possible une certaine énergie pour créer une turbulence.

M. DODU. - Il me semble qu'il y a deux problèmes de stabilité.

D'abord un problème de stabilité longitudinale qui paraît résolu. Qu'en est-il de la longueur et de la dimension transversale des cellules. Si la dimension transversale était grande, le phénomène resterait-il encore stable.

D'autre part, il semble que dans le fonctionnement de cet appareil, les débits successifs de chaque motif sont aussi stables, en ce sens que les mêmes quantités de liquides se répartissent également sur une verticale. Est-ce qu'on n'observe pas des montées et des descentes de liquide ? Est-ce une stabilité naturelle?

M. BELIN. - Votre première question concerne la longueur des cols. M. BOLDO a demandé à ses successeurs d'explorer la corrélation qui peut exister entre le régime hydrodynamique et son transfert vis-à-vis de la longueur des cols. Pour le présent, seules les extrémités peuvent modifier les courbes.

Dans la maquette, la longueur de col était de $80 \mathrm{~mm}$ et les effets de bord étaient les mêmes que sur cet appareil où la longueur du col est de $500 \mathrm{~mm}$. Cela pourrait fausser la place des courbes liquides. Mais la phénoménologie de l'aire interfaciale est simplement décalée par homothétie.

Au niveau industriel, il existe des motifs qui vont jusqu'à $1,75 \mathrm{~m}$ et on n'a jamais eu aucun problème de décalage de rendement ou d'efficacité par rapport aux maquettes qu'on avait faites en première approche.

Votre deuxième question concerne la stabilité verticale. A ce sujet, on retombe dans le problème suivant : quel est l'appareil qui donne l'énergie et comment la donne-t-il ?

C'est le choix du rapport pression dynamique/pression statique qui est primordial. Pour une pression dynamique correctement choisie, le laveur est stable naturellement. Les remplissages maximum atteints sont de 20 cellules.

M. BONNIN. - Etes-vous allé jusqu'à essayer, pour les débits de gaz, de bloquer complètement le débit liquide vers le bas, pour empêcher le liquide de descendre ?

M. BELIN. - C'est la définition de l'engorgement telle que l'a faite M. BOLDO ; il l'a montrée dans son film ; c'est-à-dire avoir une accélération des gouttes au niveau de chaque étage telle qu'elles puissent franchir le gradient décroissant de vitesse lors de l'élargissement du divergent de façon à avoir encore assez d'énergie résiduelle pour passer d'un étage inférieur à un étage supérieur. Il y a un phénomène de rétention augmentant du bas vers le haut et le blocage.

Ensuite, le problème est de savoir à quelle hauteur du liquide je pourrais vaincre la pression statique de l'appareil ; sinon, au bout d'un moment, le liquide redescend.

J'ai fait des essais de ce genre dans un autre appareil, avec une dynamique faible, et j'ai réussi à faire chuter le débit gazeux jusqu'à pratiquement 0 à perte de charge constante en augmentant le débit liquide. Il y avait alors encombrement total de la colonne par le liquide. 


\section{Abstract}

Hydrodynamics and mass transfer in a multicellular gas/liquid contactor

The Techfina multicell scrubber is a new gas-liquid contactor that is presented as a regular package in three directions of elementary cells in which the gas and the liquid phases are flowing countercurrently and vertically.

The results of experiments carried out first in an elementary cell, with a $80 \mathrm{~mm}$ length, $80 \mathrm{~mm}$ wideness, $20 \mathrm{~mm}$ heigth having a $10 \mathrm{~mm}$ distance at the throat and then in packages of several such cells ( 1 to 9 ) are reported.

The hydrodynamics study shows that when the liquid flowrate $L$ is kept constant while the gas flowrate $G$ is progressively increased the following flow regimes or patterns are observed : trickling flow, surging flow, picking up the liquid by the gas, then loading and flooding. In the flow regime diagram $L-G$ the operating zone is defined for the two-phase flowrates corresponding to an hydrodynamic operation comprised between the "picking up" point and the flooding (figure 3 ).

Typical variations of the pressure drop with the gas flowrates for constant liquid flowrates in an elementary cell are presented in Figure 4. It is observed that the curves always show a discontinuity corresponding to the picking up pheno- menum. The same trend is observed for the liquid holdup. The additivity of the pressure drop and liquid holdup in the case of the package of several elementary cells has been checked. Moreover it has been possible to correlate the liquid holdup with the values of the pressure drop corresponding to the same gas velocity at the throat of the elementary cell. This pressure drop is defined as the corrected pressure drop.

The mass transfer study leads to the presentation of the performances of the scrubber characterized by the efficiency and the Number of Transfer Unit, NUT. The absorption of dilute $\mathrm{SO}_{2}$ in air in to aqueous $\mathrm{NaOH}$ solutions is used in the conditions of an instantaneous surface and irreversible reaction totally controlled by the mass transfer resistance in the gas phase. For an elementary cell the efficiency is approximatively comprised between 50 and $60 \%$ (Figure 6). It has been possible to correlate the variations of NUT with the corrected pressure drop for one and several elementary cells. Experimental data show that the additivity of the performances of the cells is not satisfied because of the hydrodynamics phenomenum of liquid entrainment (priming). 